\title{
The Importance of Spatial Information in Sunspot Records
}

John M. Brooke

MRCCS, Manchester Computing, University of Manchester, Oxford Road, Manchester, M13 9PL, United Kingdom

Jaan Pelt

Tartu Observatory, Tõravere, 61602, Estonia

Pentti Pulkkinen

Academy of Finland, P.O. Box 99, 00501 Helsinki, Finland

Ilkka Tuominen

Astronomy Division, University of Oulu, P.O. Box 333, 90571 Oulu, Finland

\begin{abstract}
We show that the spatial information in historical sunspot records, in particular the latitude of the spots, can be of great importance in determining the long term behaviour of the solar magnetic cycle. We examine a continuous record of sunspots with full spatial data from 18531996 compiled from the records of Carrington, Spörer, the GPR and SOON. Analysis of the latitudinally-weighted observations indicates that there is a previously unremarked oscillation of the solar magnetic equator with a period of approximately 90 years.
\end{abstract}

\section{Introduction}

Since the discovery by Schwabe in 1843 of the cyclic nature of sunspot activity, there has been a great deal of attention paid to the nature of these cycles. In 1849 Wolf established a measure of sunspot activity, the Wolf number, defined as $W=k(10 g+f)$ where $g$ is the number of sunspot groups, $f$ is the total number of spots and $k$ is a factor allowing comparison between different observation series. Based on this measure we have the Zurich series of Wolf numbers which extends back to 1749. Since the counts for $W$ are taken over the whole visible surface of the Sun, all information about the spatial distribution of the sunspot activity is lost. Realising the importance of such spatial information, Carrington in 1853 began the recording of the latitudes and longitudes of spots and this eventually resulted in the Greenwich series of photographic recordings of the solar surface which lasted from 1874 to 1976 . Maunder introduced the famous Maunder or butterfly diagram (so called because the pattern of the cycles resembles the wings of a butterfly) which seems to show spot activity as a travelling wave beginning in the higher latitudes and proceeding to the equator. 

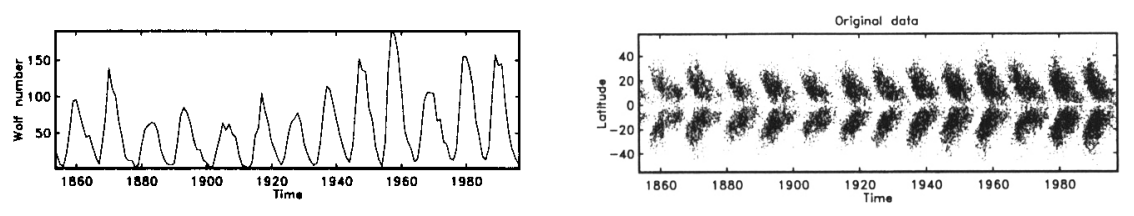

Figure 1. Two contrasting methods of showing the Schwabe cycle: left; Wolf number vs time 1853-1996, right; Maunder diagram showing spot latitude vs time $1853-1996$.

A comparison between the one-dimensional and two-dimensional representations of the solar cycle can be seen in Figure 1. A central point of our analysis is that these representations of the Schwabe cycle are not equivalent; the Wolf number representation loses all information about the spatial location of the spots. One consequence can be seen immediately from visual inspection of Figure 1 , in the Wolf number series the cycle minima are not precisely defined and are a perceptible distance from zero. In the Maunder diagram it is clear that there is a void region between each cycle, however the time intervals for this zero occurrence of sunspots vary progressively with latitude. It is now clear why the Wolf number series does not show precise minima very close to zero; spots from adjacent cycles are counted together, the new spots at high latitudes and the older spots near the equator.

\section{The data sets and methods}

We use the sunspot data over the whole range of modern observations, starting from Carrington's (1853-1861) and Spörer's (1861-1894) data. To extend this record to the present day (i.e. 1874-1996), the data from the Greenwich Photoheliographic Results and the Solar Optical Observing Network have been combined $^{1}$. These data sets cover completely sunspot cycles 10 to 22 of which cycles 10 and 11 are taken from Carrington/Spörer data and cycles 12 to 22 from the Greenwich data. This long-term data have been used to analyse solar photospheric velocities by Pulkkinen \& Tuominen (1998) where the data are also introduced in more detail.

It is important to note that before Carrington's pioneering observations there were no systematic records of the spatial distributions of the sunspots apart from the observations from France in the latter part of the 17th and early part of the 18th century, Ribes \& Nesme-Ribes (1993).

\section{Oscillations of the solar magnetic equator}

It is known that the distribution of sunspots between the northern and southern hemispheres is asymmetric in the short time scale and may undergo longer and more systematic variation (as discussed above). The usual index of sunspot

\footnotetext{
${ }^{1}$ See http://wwwssl.msfc.nasa.gov/ssl/pad/solar/greenwch.htm for the 1874-1996 data.
} 


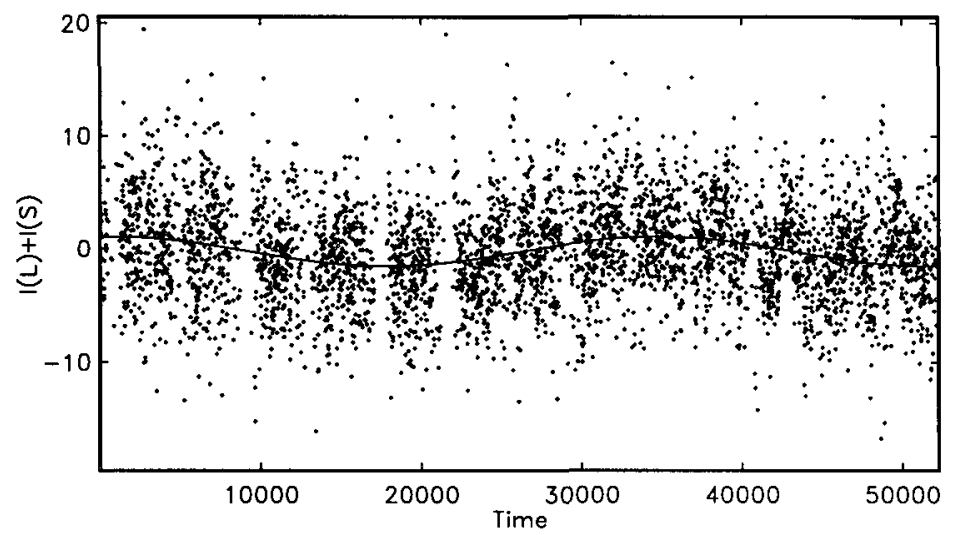

Figure 2. Variation of the magnetic equator of the sunspots. See text for full explanation.

asymmetry is $A S=(N-S) /(N+S)$ where $N$ and $S$ are the total activity counts in the northern and southern hemispheres. Such a measure loses all information about the latitudinal variation of the sunspots and from Figure 1 it can be seen that the latitudinal distribution is an important component of the Schwabe cycle.

As the mean latitude of sunspots, or the whole sunspot belt, is varying between cycles and hemispheres, their relative distance from the equator is changing too. This can be seen, when we calculate the latitude of the "magnetic equator" defined by sunspot latitudes. This is defined as the sum of the sunspot latitudes. The latitude is a signed quantity $(\langle\lambda(S)\rangle$ is negative) and we can form an average mean latitude of the spots in either hemisphere at a given time. We then define the magnetic equator as $M=\langle\lambda(N)\rangle+\langle\lambda(S)\rangle$.

An interesting pattern is seen in Figure 2 where this sum is plotted as 10day averages with an 11 point smoothing filter. In a symmetric case this sum should be zero, but this band, although wide, is clearly moving up and down rather systematically. If this is fitted to a sinusoidal profile (solid line in Figure 2 ), we get a variation in the magnetic equator $M$

$$
M=1.29( \pm 0.10) \cos (2 \pi t / P)+0.20( \pm 0.21) \sin (2 \pi t / P),
$$

where time $t$ is measured in days, $t=0$ being Nov 8,1853 , the first measurement by Carrington. The period of this variation $P=33900 \pm 950$ days, and the amplitude $1.31 \pm 0.13$ degrees. For further detailed analysis see Pulkinnen et al. (1999) where it is demonstrated that both the period and the form of the oscillation is robust with respect to the averaging interval. Thus we consider it to be a genuine effect.

\section{A new method of defining cycle length}

The void regions referred to in Section 1 can be used to identify the start of each cycle precisely and thus get a robust definition of cycle length. Details 

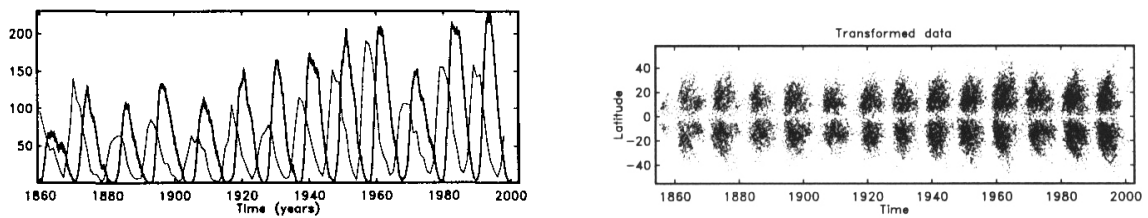

Figure 3. Cycles based on nonlinear transformation of the time axis of Figure 1, following the onset of activity as a travelling wave. Left: Wolf number (1853-1996), thick line is transformed data, thin line is original data, right: latitude vs redefined time (1853-1996). It can be seen how the cycles are clearly separated and the minima in Wolf number are more precisely defined.

of the method are in Pelt et al. (2000) but the results can be seen in Figure 3. Essentially we perform a nonlinear shift of the time axis of the Maunder diagram to make the edge of onset of activity the new time axis. Thus we follow the onset of activity of a travelling wave. If we reconstruct the Wolf number diagram with this new "time" axis (strictly speaking it is an axis of constant phase of the travelling wave) we see that the minima are now more precisely defined and very close to zero. This is because we have separated the cycles in our counting procedure. From this we can demonstrate that there is a periodic change in cycle length which coincides with the oscillations of the solar magnetic equator. This establishes the Gleissberg cycle (Gleissberg 1967) as an oscillation in equatorial symmetry and cycle length with a period of approximately 90 years. These methods of spatial analysis have been developed very recently and there is every hope that more information about the solar cycle can be derived from the historical records. This will provide important currents for nonlinear dynamo models of the solar cycle.

\section{References}

Gleissberg, W. 1967, Solar Phys. 2, 231

Pelt, J., Brooke, J.M., Pulkkinen, P., \& Tuominen, I. 2000, to appear in A\&A Pulkkinen, P., \& Tuominen, I. 1998, A\&A, 332, 748

Pulkinnen, P., Brooke, J.M., Pelt, J., \& Tuominen, I. 1999, A\&A, 341, L43

Ribes, J.C., \& Nesme-Ribes, E. 1993, A\&A 276, 549

Royal Observatory, Greenwich: 1874-1976, Greenwich Photoheliographic Results, in 103 volumes

Spörer, G. 1884, Publ. Astrophys. Obs. Potsdam, 10, 1

Wolf, R. 1861, Astr. Mitt. Eidg. Sternwarte Zürich, No 8 